\title{
Stability of Fibonacci Functional Equation
}

Sandeep* and Sushma**

A.I.J.H.M college Rohtak*

Kanya Mahavidayalaya Kharkhoda, Sonepat**.

E-mail : lathersushma@yahoo.com

Abstract. In this paper, we solve the Fibonacci functional equation, $f(x)=f(x-1)+f(x-2)$ and discuss its generalized Hyers-Ulam-Rassias stability in Banach spaces and stability in Fuzzy normed space.

Indexing terms/Keywords: Fibonacci Functional Equation

Language: English

Date of Submission: 2018-01-27

Date of Acceptance: 2018-02-20

Date of Publication: 2018-03-01

ISSN: 2347-1921

Volume: 14 Issue: 01

Journal: Journal of Advances in Mathematics

Publisher: CIRWORLD

Website: https://cirworld.com

This work is licensed under a Creative Commons Attribution 4.0 International License. 


\section{Introduction.}

A question in the theory of functional equations is the following "When is it true that a function which approximately satisfies a functional equation $\in$ must be close to an exact solution $\in$ ?" If the problem accepts a solution, we say that the equation $\in$ is stable.

In 1940, S.M. Ulam [8] gave a wide-ranging talk before the Mathematics Club of the University of Wisconsin in which he discussed a number of important unsolved problems. Among those was the following question concerning the stability of homomorphism:

Let $\left(\mathrm{G}_{1},{ }^{*}\right)$ be a group and $\left(\mathrm{G}_{2}, \circ, \mathrm{d}\right)$ be a metric group with the metric $\mathrm{d}$. Given $\in>0$, does there exists a $\delta_{\epsilon}>0$ such that if a mapping $\mathrm{h}: \mathrm{G}_{1} \rightarrow \mathrm{G}_{2}$ satisfies the inequality $\mathrm{d}\left(\mathrm{h}\left(\mathrm{x}^{*} \mathrm{y}\right), \mathrm{h}(\mathrm{x}) \circ \mathrm{h}(\mathrm{y})\right)<\delta_{\in} \forall \mathrm{x}, \mathrm{y} \in \mathrm{G}_{1}$, then there is a mapping $H: G_{1} \rightarrow G_{2}$ such that for each $x, y \in G_{1} H\left(x^{*} y\right)=H(x) \circ H(y)$ and $d(h(x), H(x))<\in$ ?

In the next year, D. H. Hyers [3], gave answer to the above question for additive groups under the assumption that groups are Banach spaces. In 1978, T. M. Rassias [7] proved a generalization of Hyers' theorem for additive mapping as a special case in the form of following result.

Suppose that $E$ and $F$ are real normed spaces with $F$ a complete normed space, $f: E \rightarrow F$ is a mapping such that for each fixed $x \in E$ the mapping $t \rightarrow f(t x)$ is continuous on $R$, and let there exist $\in \geq 0$ and $p \in[0,1)$ s.t

$$
\|f(x+y)-f(x)-f(y)\| \leq \varepsilon\left(\|x\|^{p}+\|y\|^{p}\right) \mathrm{x}, \mathrm{y} \in \mathrm{E} .
$$

Then there exists a unique linear mapping $\mathrm{T}: \mathrm{E} \rightarrow \mathrm{F}$ s.t $\|f(x)-T(x)\| \leq \varepsilon \frac{\|x\|^{p}}{\left(1-2^{p-1}\right)}, \mathrm{x} \in \mathrm{E}$.

In this paper we discuss the stability of Fibonacci functional equation

$f(x)=f(x-1)+f(x-2)$.

A function $f: R \rightarrow X$ will be called a Fibonacci functional equation if it satisfies (1), for all $x \in R$, where $X$ is a real vector space. By $\alpha$ and $\beta$ we denote the positive and negative roots respectively of the quadratic equation $x^{2}-x-$ $1=0$. i.e., $\alpha=\frac{1+\sqrt{5}}{2}$ and $\beta=\frac{1-\sqrt{5}}{2}$ for any $x \in R$. M. M. Parizi and M. E. Gordji [11] proved the stability of Fibonacci functional equation in Modular functional spaces. S. M. Jung [10] also proved the stability of Fibonacci functional equation in real Banach space as following:

Theorem1: Let $(X,\|\|$.$) be a real Banach space. If a function f: R \rightarrow X$ satisfies the inequality, $\|f(x)-f(x-1)-f(x-2)\| \leq \epsilon$

for all $x \in R$ and for some $\epsilon>0$, Then there exists a Fibonacci function $F: R \rightarrow X$ such that $\|f(x)-F(x)\| \leq\left(1+\frac{2}{\sqrt{5}}\right) \varepsilon$

for all $x \in R$.

Proof. We get from (1.1),

$\|f(x)-\alpha f(x-1)-\beta[f(x-1)-\alpha f(x-2)]\| \leq \epsilon$,

For each $x \in R$. If we replace $x$ by $x-k$ in (1.3), then we have,

$\|f(x-k)-\alpha f(x-k-1)-\beta[f(x-k-1)-\alpha f(x-k-2)]\| \leq \epsilon$

And

$\left\|\beta^{k}[f(x-k)-\alpha f(x-k-1)]-\beta^{k+1}[f(x-k-1)-\alpha f(x-k-2)]\right\| \leq \beta^{k} \epsilon$ 
Thus, we have,

$\left\|f(x)-\alpha f(x--1)-\beta^{n}[f(x-n)-\alpha f(x-n-1)]\right\| \leq \sum_{k=0}^{n-1}|| \beta^{k}[f(x-k)-\alpha f(x-k-1)]-\beta^{k+1}[f(x-k-1)-\alpha f(x-k-2)]|| \leq$ $\sum_{k=0}^{n-1}|\beta|^{k} \epsilon$

From (1.4), we get $\left\{\beta^{k}[f(x-n)-\alpha f(x-n-1)\}\right.$ is a Cauchy sequence. Therefore, we can define a function $F_{1}: R \rightarrow X$ by $\mathrm{F}_{1}=\lim _{n \rightarrow \infty} \beta^{k}[f(x-n)-\alpha f(x-n-1)]$, since $\mathrm{X}$ is complete so $\mathrm{F}_{1}$ is in $\mathrm{X}$. We obtain that

$$
\begin{aligned}
\mathrm{F}_{1}(\mathrm{x}-1)+\mathrm{F}_{1}(\mathrm{x}-2) & =\beta^{-1} \lim _{n \rightarrow \infty} \beta^{n+1}[f(x-n-1)-\alpha f(x-n)] \\
& +\beta^{-2} \lim _{n \rightarrow \infty} \beta^{n+2}[f(x-(n+2))-\alpha f(x-(n+2)-1)] \\
& =\beta^{-1} \mathrm{~F}_{1}(\mathrm{x})+\beta^{-2} \mathrm{~F}_{1}(\mathrm{x})=\mathrm{F}_{1}(\mathrm{x})
\end{aligned}
$$

For all $x \in R$. Hence $F_{1}$ is a Fibonacci function. If $n$ goes to infinity, then (1.5) implies

$\left\|f(x)-\alpha f(x-1)-F_{1}(x)\right\| \leq \frac{3+\sqrt{5}}{2} \epsilon$

For every $x \in R$.

From (1.1)

$\|f(x)-\beta f(x-1)-\alpha[f(x-1)-\beta f(x-2)]\| \leq \epsilon$,

For each $x \in R$. If we replace $x$ by $x+k$ in (1.7), then we have,

$\|f(x+k)-\beta f(x+k-1)-\alpha[f(x+k-1)-\beta f(x+k-2)]\| \leq \epsilon$

And

$\left\|\alpha^{-k}[f(x+k)-\beta f(x+k-1)]-\alpha^{-k+1}[f(x+k-1)-\beta f(x+k-2)]\right\| \leq \alpha^{-k} \epsilon$

Thus, we have,

$\| \alpha^{-n}\left[\mathrm{f}(\mathrm{x}+\mathrm{n})-\beta \mathrm{f}(\mathrm{x}+\mathrm{n}-1)-[\mathrm{f}(\mathrm{x})-\beta \mathrm{f}(\mathrm{x}-1)] \| \leq \sum_{k=0}^{n}|| \alpha^{-\mathrm{k}}[\mathrm{f}(\mathrm{x}+\mathrm{k})-\beta \mathrm{f}(\mathrm{x}+\mathrm{k}-1)]-\alpha^{-\mathrm{k}+1}[\mathrm{f}(\mathrm{x}+\mathrm{k}-1)-\beta \mathrm{f}(\mathrm{x}+\mathrm{k}-\right.$ 2)] $\mid \leq \sum_{k=0}^{n} \alpha^{-\mathrm{k} \epsilon}$

From (1.8), we get $\left\{\alpha^{-n}[f(x+n)-\beta f(x+n-1)\}\right.$ is a Cauchy sequence. Therefore, we can define a function $F_{2}: R \rightarrow X$ by $\mathrm{F}_{2}=\lim _{n \rightarrow \infty} \alpha^{-n}[f(x+n)-\beta f(x+n-1)]$, since $\mathrm{X}$ is complete so $\mathrm{F}_{2}$ is in $\mathrm{X}$. We obtain that

$$
\begin{aligned}
\mathrm{F}_{2}(\mathrm{x}-1)+\mathrm{F}_{2}(\mathrm{x}-2)= & \alpha^{-1} \lim _{n \rightarrow \infty} \alpha^{-(n-1)}[f(x+n-1)-\beta f(x+n-1)] \\
& \left.+\alpha^{-2} \lim _{n \rightarrow \infty} \alpha^{-n+2}[f(x+n-2))-\beta f(x+(n-2)-1)\right] \\
& =\alpha^{-1} \mathrm{~F}_{2}(\mathrm{x})+\alpha^{-2} \mathrm{~F}_{2}(\mathrm{x})=\mathrm{F}_{2}(\mathrm{x})
\end{aligned}
$$

For all $x \in R$. Hence $F_{2}$ is a Fibonacci function. If $n$ goes to infinity, then (1.9) implies

$\left\|F_{2}(x)-f(x)+\beta f(x-1)\right\| \leq \frac{\sqrt{5}+1}{2} \epsilon$

For every $x \in R$. 
From (1.6) and (1.10), we have

$$
\begin{aligned}
\| f(x)-\left[\frac{\beta}{\beta-\alpha} F_{1}(x)-\frac{\alpha}{\beta-\alpha} F_{2}\right] & \left\|=\frac{1}{|\beta-\alpha|}\right\|(\beta-\alpha) f(x)-\left[\beta F_{1}(x)-\alpha F_{2}(x)\right] \| \\
& \leq \frac{1}{\alpha-\beta}\left\|\beta f(x)-\alpha \beta f(x-1)-\beta F_{1}(x)\right\|+\frac{1}{\alpha-\beta}\left\|\alpha F_{2}(x)-\alpha f(x)+\alpha \beta f(x-1)\right\| \\
& \leq\left(1+\frac{2}{\sqrt{5}}\right) \epsilon
\end{aligned}
$$

For all $x \in R$. Now we set

$\mathrm{F}(\mathrm{x})=\frac{\beta}{\beta-\alpha} F_{1}(x)-\frac{\alpha}{\beta-\alpha} F_{2}$

Clearly $F(x)$ is the Fib0nacci function.

Now we prove the stability of Fibonacci functional equation in fuzzy normed space.

Theorem 2: Let $(X, N)$ and $\left(Y, N^{\prime}\right)$ be fuzzy normed spaces. If $f: R \rightarrow X$ satisfies the inequality

$N(f(x)-f(x-1)-f(x-2), t) \geq N^{\prime}(\phi(x), t)$

for all $x \in R$, then there exists a Fibonacci function $F: R \rightarrow X$ such that

$N(f(x)-F(x)) \geq N^{\prime}\left(\phi(x),\left(1+\frac{2}{\sqrt{5}}\right) t\right)$.

Proof. We get from (2.1),

$N(f(x)-\alpha f(x-1)-\beta[f(x-1)-\alpha f(x-2)], t) \geq N^{\prime}(\phi(x), t)$

For each $x \in R$. If we replace $x$ by $x-k$ in (2.2), then we have,

$N(f(x-k)-\alpha f(x-k-1)-\beta[f(x-k-1)-\alpha f(x-k-2)], t) \geq N^{\prime}(\phi(x-k), t)$

And

$N\left(\beta^{k}[f(x-k)-\alpha f(x-k-1)]-\beta^{k+1}[f(x-k-1)-\alpha f(x-k-2)], \beta^{k} t\right) \geq N^{\prime}(\phi(x-k), t)$

Thus, we have,

$N\left(f(x)-\alpha f(x-1)-\beta^{n}[f(x-n)-\alpha f(x-n-1)], \sum_{k=0}^{n-1}|\beta|^{k} t\right) \geq \min \left\{N\left(\beta^{k} f(x-k)-\alpha f(x-k-1)-\beta^{k+1}\left[f(x-k-1)-\alpha f(x-k-2), \beta^{k} \quad t\right), k=0,1, \ldots . . n-\right.\right.$ $1\} \geq \mathrm{N}^{\prime}\left(\phi(\mathrm{x}), \sum_{k=0}^{n-1}|\beta|^{k} \mathrm{t}\right)$

From (2.4), we get $\left\{\beta^{n}[f(x-n)-\alpha f(x-n-1)\}\right.$ is a Cauchy sequence. Therefore, we can define a function $F_{1}: R \rightarrow X$ by

$\mathrm{F}_{1}=\lim _{n \rightarrow \infty} \beta^{k}[f(x-n)-\alpha f(x-n-1)]$, since $\mathrm{X}$ is complete so $\mathrm{F}_{1}$ is in $\mathrm{X}$. We obtain that

$$
\begin{aligned}
\mathrm{F}_{1}(\mathrm{x}-1)+\mathrm{F}_{1}(\mathrm{x}-2)= & \beta^{-1} \lim _{n \rightarrow \infty} \beta^{n+1}[f(x-n-1)-\alpha f(x-n)] \\
& +\beta^{-2} \lim _{n \rightarrow \infty} \beta^{n+2}[f(x-(n+2))-\alpha f(x-(n+2)-1)] \\
& =\beta^{-1} \mathrm{~F}_{1}(\mathrm{x})+\beta^{-2} \mathrm{~F}_{1}(\mathrm{x})=\mathrm{F}_{1}(\mathrm{x})
\end{aligned}
$$

For all $x \in R$. Hence $F_{1}$ is a Fibonacci function. If $n$ goes to infinity, then (2.4) implies

$N\left(f(x)-\alpha f(x-1)-F_{1}(x), t\right) \geq N^{\prime}\left(\phi(x),\left(\frac{3+\sqrt{5}}{2}\right) t\right)$ 
For every $x \in R$.

From (2.1)

$N(f(x)-\beta f(x-1)-\alpha[f(x-1)-\beta f(x-2)], t) \geq N^{\prime}(\phi(x), t)$,

For each $x \in R$. If we replace $x$ by $x+k$ in (2.6), then we have,

$N(f(x+k)-\beta f(x+k-1)-\alpha[f(x+k-1)-\beta f(x+k-2)], t) \geq N^{\prime}(\phi(x+k), t)$

And

$N\left(\alpha^{-k}[f(x+k)-\beta f(x+k-1)]-\alpha^{-k+1}[f(x+k-1)-\beta f(x+k-2)], \alpha^{-k} t\right) \geq N^{\prime}(\phi(x+k), t)$

Thus, we have,

$\left.\mathrm{N}\left(\alpha^{-n}\left[\mathrm{f}(\mathrm{x}+\mathrm{n})-\beta \mathrm{f}(\mathrm{x}+\mathrm{n}-1)-[\mathrm{f}(\mathrm{x})-\beta \mathrm{f}(\mathrm{x}-1)], \sum_{k=0}^{n-1} \alpha^{-k} \mathrm{t}\right)\right]\right)$

$\geq \min \left\{N\left(\alpha^{-k} f(x+k)-\beta f(x+k-1)-\alpha^{-k+1}\left[f(x+k-1)-\beta f(x+k-2), \alpha^{-k} t\right), k=0,1, \ldots . n-1\right\}\right.$

$\geq \mathrm{N}^{\prime}\left(\phi(\mathrm{x}),\left.\sum_{k=0}^{n-1} \alpha\right|^{-k} \mathrm{t}\right)$

From (2.7), we get $\left\{\alpha^{-n}[f(x+n)-\beta f(x+n-1)\}\right.$ is a Cauchy sequence. Therefore, we can define a function $F_{2}: R \rightarrow X$ by $\mathrm{F}_{2}=\lim _{n \rightarrow \infty} \alpha^{-n}[f(x+n)-\beta f(x+n-1)]$, since $\mathrm{X}$ is complete so $\mathrm{F}_{2}$ is in $\mathrm{X}$. We obtain that

$$
\begin{aligned}
\mathrm{F}_{2}(\mathrm{x}-1)+\mathrm{F}_{2}(\mathrm{x}-2)= & \alpha^{-1} \lim _{n \rightarrow \infty} \alpha^{-(n-1)}[f(x+n-1)-\beta f(x+n-1)] \\
& \left.+\alpha^{-2} \lim _{n \rightarrow \infty} \alpha^{-n+2}[f(x+n-2))-\beta f(x+(n-2)-1)\right] \\
& =\alpha^{-1} \mathrm{~F}_{2}(\mathrm{x})+\alpha^{-2} \mathrm{~F}_{2}(\mathrm{x})=\mathrm{F}_{2}(\mathrm{x}),
\end{aligned}
$$

For all $x \in R$. Hence $F_{2}$ is a Fibonacci function. If $n$ goes to infinity, then (2.8) implies

$N\left(F_{2}(x)-f(x)+\beta f(x-1), t\right) \geq N^{\prime}\left(\phi(x),\left(\frac{\sqrt{5}+1}{2}\right) t\right)$

For every $x \in R$.

From (2.5) and (2.9), we have

$\mathrm{N}\left(\mathrm{f}(\mathrm{x})-\left[\frac{\beta}{\beta-\alpha} F_{1}(x)-\frac{\alpha}{\beta-\alpha} F_{2}\right], \mathrm{t}\right)=\mathrm{N}\left((\beta-\alpha) \mathrm{f}(\mathrm{x})-\left[\beta \mathrm{F}_{1}(\mathrm{x})-\alpha \mathrm{F}_{2}(\mathrm{x})\right],|\beta-\alpha| \mathrm{t}\right)$

$$
\begin{gathered}
\geq \min \left\{N\left(\beta f(x)-\alpha \beta f(x-1)-\beta F_{1}(x), \frac{\alpha-\beta}{2} t\right), N\left(\alpha F_{2}(x)-\alpha f(x)+\alpha \beta f(x-1), \frac{\alpha-\beta}{2} t\right)\right. \\
\geq N^{\prime}\left(\phi(x),\left(1+\frac{2}{\sqrt{5}}\right) t\right)
\end{gathered}
$$

For all $x \in R$. Now we set

$\mathrm{F}(\mathrm{x})=\frac{\beta}{\beta-\alpha} F_{1}(x)-\frac{\alpha}{\beta-\alpha} F_{2}$

Clearly $F(x)$ is the Fibonacci function.

\section{References}

[1] Hyers, D.H., Isac, G. and Rassias, Th.M., On the asymptoticity aspect of Hyers-Ulam stability of mappings, Proc. Amer. Math. Soc. 126 (1998) 425-430. 
[2] Hyers, D.H., Isac, G. and Rassias, Th.M., Stability of Functional Equations in Several Variables, Birkhäuser, Basel, 1998.

[3] Hyers, D.H., On the stability of the linear functional equation, Proc. Nat. Acad. Sci. 27 (1941) 222-224.

[4] Hyers,D.H. and Rassias, Th.M. Approximate homomorphisms, Aequationes Math. 44 (1992) 125-153.

[5] Rassias, T.M. On the Stability of Functional Equations and a Problem of Ulam, Acta Appl. Math., 62(2000), 123- 130.

[6] Rassias, Th. M., On the stability of functional equations in Banach spaces, J. Math. Anal. Appl. 251 (2000) 264-284.

[7] Rassias, Th.M., On the stability of the linear mapping in Banach spaces, Proc. Amer. Math.Soc. 72 (1978) 297-300.

[8] Ulam, S.M., A Collection of the Mathematical Problems, Interscience Publ. New York, 1960.

[9] Ulam, S.M., Problems in Modern Mathematics, John Wiley \& Sons, New York, USA, 1964.

[10] S.M.Jung., Hyers-Ulam stability of Fibonacci functional equation, Bulletin of Iranian Mathematical society, 35(2),2009, 217-227.

[11] M.N.Parizi and M.E.Gordji, Hyers-Ulam stability of Fibonacci functional equation in Modular functional spaces, Journal of mathematics and computer science, 10(2014) 1-6. 